\title{
Quality of Potato Chips as Influenced by Aloe Vera Coating
}

\author{
Kashif Sarfraz Abbasi ${ }^{1}$, Tariq Masud ${ }^{2}$, Sartaj Ali $^{3, *}$, Talat Mahmood ${ }^{1}$, Azhar Hussain ${ }^{3}$, Muhammad Liaquat ${ }^{1}$, \\ Muhammad Jahangir ${ }^{1}$ \\ ${ }^{1}$ Department of Agriculture Sciences, University of Haripur, Haripur Pakistan; \\ ${ }^{2}$ Department of Food Technology, PMAS, Arid Agriculture University, Rawalpindi, Pakistan; \\ ${ }^{3}$ Department of Agriculture and Food Technology, Karakoram International University, Gilgit, Pakistan \\ *Corresponding author: sartaj_kiu@yahoo.com
}

Received December 29, 2014; Revised February 09, 2015; Accepted March 03, 2015

\begin{abstract}
The objectives of the current endeavor were to develop a healthy halal product with greater consumer acceptance level. Chips prepared from stored potatoes $\left(10 \pm 1^{\circ} \mathrm{C}\right.$ and $70 \pm 5 \%$ relative humidity) at thirty days interval were coated with different concentrations of aloe vera gel before frying. Significant variations $(p<0.05)$ were observed in the quality attributes as a result of gel concentrations and storage intervals. The coated chips were found high in moisture content with lower fat absorption and appreciable sensorial scores. While comparing the different concentrations of aloe vera, it revealed that 10 and $20 \%$ gel produced potato chips with acceptable color which can also be associated with low perceived acryl amide formation. Steady increase in oil uptake and moisture contents with reduction in sensory scores was observed along the extended storage intervals. The overall results revealed that $20 \%$ aloe vera gel proved to be the best coating for potato chips with moderate moisture contents, reduced oil uptake and appreciable sensorial scores.
\end{abstract}

Keywords: plant based coating, potato chips, aloe vera gel, halal processed products

Cite This Article: Kashif Sarfraz Abbasi, Tariq Masud, Sartaj Ali, Talat Mahmood, Azhar Hussain, Muhammad Liaquat, and Muhammad Jahangir, "Quality of Potato Chips as Influenced by Aloe Vera Coating." Journal of Food and Nutrition Research, vol. 3, no. 3 (2015): 157-161. doi: 10.12691/jfnr-3-3-5.

\section{Introduction}

Potato chips are the oil rich (35-40\%) products and considered as preferred snack foods world over due to their palatable taste and ease of preparation. High oil contents however of grave concern for the peoples suffering from chronic heart diseases and obesity. Different techniques like, modification in size and thickness [1], pre drying [2], modification in frying techniques [3], frying medium [5], frying temperature [6] and potato chips coatings [7] have been employed to minimize oil contents in the thermally processed products.

Oil uptake in the fried products is determined by two mechanisms i.e. condensation effect and capillary effect [6], which are altered by the application of different coating materials. Since the oil uptake by potato chips during frying is largely the function of its surface properties. Therefore, coating is considered as promising route for its mitigation in finished processed product. It has been previously reported that the coating with cellular derivatives cause the formation of protective layer on the surface, which eventually decreases the oil uptake in the fried products [7]. Garcia et al. [8] evaluated the surface application of cellulose derivatives in different formulations for the reduced oil uptake in fried products. He concluded that coating reduce the oil uptake by 35-
$40 \%$ with appreciable retention of different sensory attributes.

Application of different coating materials help to reduce oil uptake and improve consumer preference for the fried products. These coating materials can be thin and invisible or thick like batter [9]. Albert and Mittal [10] studied the effect of different coating materials like gelatin, gellan gum, methyl cellulose, pectin, sodium caseinate, soya protein isolates and wheat protein. He found a general reduction in oil uptake as compared to control. In addition, pre processing coating on potato products has been reported to effective to mitigate acrylamide formation in the processed products [9]. The selection of proper coating material with desirable barrier and mechanical properties are essential for premium quality product. Moreover, the application of coating material is also associated with increased moisture contents and decreased color scores in the processed product which should be kept insight for the consumer acceptance.

Currently, researchers are more interested in developing plant based coating formulations for consumer safety. A variety of plants and plant based materials are being investigated for their usefulness. Aloe vera (AV) is tropical and subtropical plant widely known to its therapeutic and medicinal properties. Its mucilage gel is extracted from leaf parenchymatic cells and predominantly used against cardiovascular diseases, ulcer, gastrointestinal and renal disorders since long [11]. Owing 
to its antibiotic and anti-inflammatory properties, it has also been considered as remedy against lethal diseases like AIDS and cancer [12]. The use of AV gel has also been increasing in cosmetic industry and known to carry antifungal activity [13]. In addition, it is increasingly used as a functional ingredient in food industries for ice creams, beverages and desserts production [14]. It is reported to carry specific barrier properties and thus used as postharvest edible coating to increase the shelf life of cherries [15] and table grapes [16].

Selection of AV gel for chips coating in the present study has been carried out as a novel technique in potato processing. The application was required for the development of plant based HALAL (permissible in Islamic jurisprudence) coating material to get reduced fat uptake in potato chips along with appreciable retention of sensorial attributes. Such high quality processed product would equally be appreciated by the processor and consumer due to added economic and health benefits. In addition, prolonged storage study would also be helpful to identify storage related changes in the quality attributes of potato chips during processing.

\section{Materials and Methods}

\subsection{Material}

Potato variety "Lady Rosetta" was harvested from Potato Research Institute, Sahiwal (Punjab, Pakistan) in January 2012. The experimental material was shifted to the Postharvest Technology Lab. Food Technology Department, PMAS-Arid Agriculture University, Rawalpindi. Potatoes were washed, sorted and graded into homogenous lots and subsequently cured for one week at temperatures between $15-20^{\circ} \mathrm{C}$. After curing, the tubers were given hot water treatment $\left(55 \pm 2^{\circ} \mathrm{C}\right.$ for 15 minutes) followed by Clove Oil application (1\%) to prevent sprouting. Tubers were packed in polypropylene packaging and then stored at $10 \pm 1^{\circ} \mathrm{C}$ and $70 \pm 5 \%$ R.H. $500 \mathrm{~g}$ potato tubers were placed in each replication and 1.5 $\mathrm{kg}$ tubers were maintained in each treatment for quality evaluation at given intervals. $15 \mathrm{Kg}$ potatoes were left in each treatment, which were subjected to coating and subsequent processing at thirty days intervals.

\subsection{Aloe Vera Coating}

Pre processing potato chip coating was carried out in different concentrations of AV gel followed by subsequent frying. The gel was extracted from freshly harvested AV leaves and blanched at $70^{\circ} \mathrm{C}$ for 10 minutes. AV gel was prepared in three different formulations each with $1 \%$ sorbitol (Sigma-Aldrich, USA) added as plasticizer along with distilled water as under:

$$
\begin{array}{ll}
\mathrm{T}_{1}=\text { Control } & \mathrm{T}_{2}=\mathrm{AV}(10 \%) \\
\mathrm{T}_{3}=\operatorname{AV}(20 \%) & \mathrm{T}_{4}=\operatorname{AV}(30 \%)
\end{array}
$$

Peeled tubers were sliced (1.2-1.5 mm thick) and blanched in $1.5 \% \mathrm{NaCl}_{2}$ solution at $85^{\circ} \mathrm{C}$ for two minutes. The potato chips were dipped in prepared gel formulations for five minutes, allowed to drip off and dried before frying. After pre drying, the chips were fried in electric fryer at $180-185^{\circ} \mathrm{C}$ for three minutes using palm oil. The potato chips coating and subsequent frying were carried out at thirty days interval during the potato storage.

\subsection{Physico-Chemical Evaluation}

\subsubsection{Chips Moisture Contents}

The fried chip were cooled and placed in Dry Oven at $105^{\circ} \mathrm{C}$ till constant weight for estimation of chip moisture contents (CMC) according to AOAC [17].

$$
\mathrm{CMC}(\%)=\frac{\text { Wtbefore drying }-\mathrm{Wt} \text { after drying }}{\text { Wt before drying }} \times 100
$$

\subsubsection{Chip Oil Uptake}

The chip oil uptake (COU) in different samples was determined by Soxhlet extraction apparatus as described by AOAC [17]. Briefly, $10 \mathrm{~g}$ sample of dried chips were placed in a thimble and extracted with hexane (B.P 40$60^{\circ} \mathrm{C}$ ) in a pre weighed Soxhlet flask. The solvent was recovered after the completion of extraction process and oil containing flask was dried in oven till constant weight. The oil contents were gravimetrically determined by the following equation:

$$
\operatorname{COU}(\%)=\frac{\text { Wt. of flask }+ \text { oil }- \text { Wt. of empty flask }}{\text { Weight of sample }} \times 100
$$

\subsubsection{Chip Color}

The color of the potato chips was measured as L-value using a Minolta colorimeter (CR 300 series Japan). Lvalue corresponds to the darkness or lightness using Hunter scale (0-black, 100- white) in a potato chip which is of major commercial significance. Fifteen potato chips were evaluated per treatment and three readings were estimated per chip by rotating them at angle of $180^{\circ}$. Black and white plates were used for the colorimeter calibration.

\subsubsection{Sensory Evaluation}

Twenty five students including the faculty members of Department of Food Technology who were the habitual consumers of the potato chips were selected as judge for sensory evaluation. The judges were requested to record their degree of preferences for Crispiness (CRP) and Flavor (FLV) according to the five point hedonic scale as described by Kita [18] with few modifications.

\subsection{Statistical Analysis}

Data obtained as mean of three replications were statistically analyzed by two factor factorial in Completely Randomized Design (CRD) as described by Steel et al. [19] using M-STAT-C statistical software.

\section{Results and Discussion}

\subsection{Chip Moisture Contents}

In general pre processing application of different concentrations of AV on the potato chip showed increase in chip moisture contents (CMC). The effect of treatments, storage intervals and their interaction was found significant for the chip moisture contents $(\alpha-0.05)$. The 
increase in CMC in AV 10\%, 20\% and 30\% remained around 8.1-folds, 9-folds and 11.1-folds after storage for 180 days. In contrast, the increase in control remained less than 2-folds during the same storage period (Figure 1).

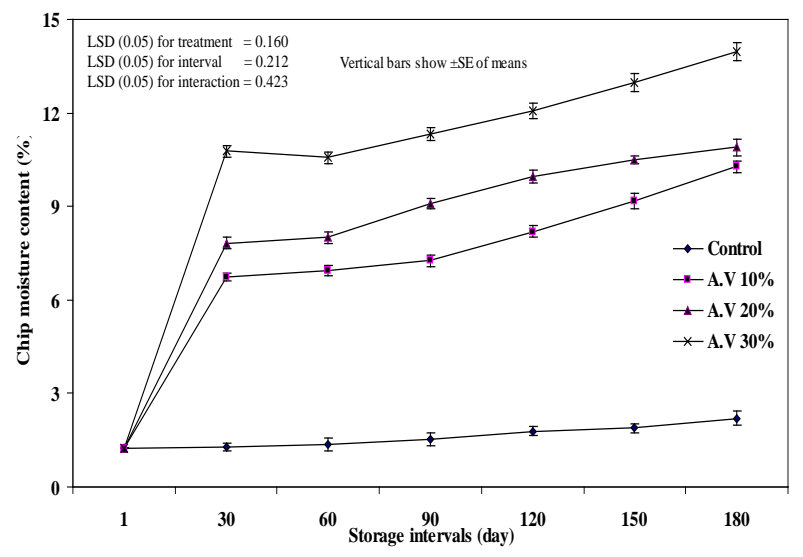

Figure 1. Variation in moisture contents in aloe vera coated chips processed from stored potatoes

Surface properties of frying raw material are very important to establish eventual fat content absorbed during processing. Modification in the surface coatings may be carried out by the application of different edible coating which can be transparent or thick like batter [6]. In doing so, uniform coating configuration on the surface is imperative to bound mass transfer during processing [20]. Garmakhany et al. [21] evaluated quality attributes of potato chips in response to different hydrocolloid applications. Different coating materials i.e. carboxymethyl cellulose, xanthan gum and guar were applied in selected concentrations before processing. He concluded that all the coating materials retained comparatively high moisture contents and reduced fat absorption as compare to control. Our results showed that increase in $\mathrm{AV}$ concentrations resulted in substantial increase in CMC after processing and a steady increase was observed along the extended storage intervals. The possible reason might be due to the barrier properties offered by the coating concentrations to mass transfer during processing [8]. Steady increase in CMC along the storage intervals in all treatments might be due to conversion of starch into soluble sugars under prolonged storage [22]. The appropriate concentration of coating material however must be established in order to prevent sogginess and reduced sensorial scores as happened in $30 \%$ AV concentrations during most of the storage period. Among the applied concentrations of AV, 20\% presented moderate CMC along with considerable decline in their oil absorption during most of the storage period. Similar observations were reported by Khalil [23] regarding increased moisture contents in French fries in response to increased coating concentrations.

\subsection{Chip Oil Uptake}

Chip oil uptake (COU) decreased in response to all coating applications during storage except in control (Figure 2). The effect of treatments, storage intervals and their interaction was found significant $(\alpha-0.05)$ for the chip oil uptake. In general different concentrations of AV showed significant reduction in COU along the storage period. The percent reduction in $\mathrm{COU}$ on $20^{\text {th }}$ day remained around $13.4 \%, 20.5 \%$ and $23.1 \%$ in AV $10 \%$, $20 \%$, and $30 \%$ respectively, in contrast, a slight increase in COU observed in control. While comparing different storage intervals, COU increased among all the treatments with lowest COU observed in 30\% AV (27.53\%) followed by $20 \%$ AV (27.98\%) and maximum COU was estimated in control $(37.00 \%)$ on $180^{\text {th }}$ day storage.

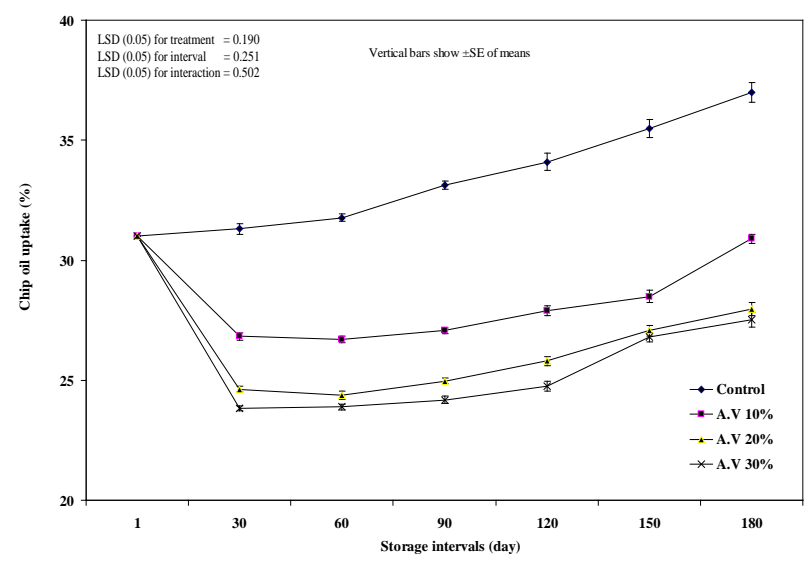

Figure 2. Changes in oil uptake in aloe vera coated chips processed from stored potatoes

Oil is the major source of flavor enhancer in potato chips. Its high level is however of great concern for the producer and consumer for economic and food safety point of view respectively. Hydrocolloid application has been carried out by different researchers to reduce the fat absorption in processed food products to address problems like obesity and coronary heart disorders [6]. The efficiency of these coating materials however depends on their barrier properties, applied concentration and ability to produce quality finished products [8]. In general, COU is thought to be associated with the moisture loss during frying and defines the volume of oil pockets in the finished product. Thus the response of COU to different concentrations of AV was found inversely proportional to their CMC during the storage period. Our results in the present study showed significant oil reduction in the processed products in response to increase in aloe vera concentrations. Minimum COU was observed in AV 30\% closely followed by AV $20 \%$ might be due to the conferred variable barrier properties by different levels of coating applications. Similar observations were recorded by Rimac et al. [24] and Albert and Mittal [10] regarding the reduction in oil uptake with the increase in coating concentrations. In addition, steady increase in COU was observed along the storage period in control which might be due to inverse relationship between COU and tuber specific gravity at the twilight of storage period [25].

\subsection{Chip Color}

Chip color (CCL) estimated as approximate L-value generally decreased with the increase in storage duration. The effect of treatments, storage intervals and their interaction was found significant $(\alpha-0.05)$ for the chip color. L-value decreased in all treatments with the increase in storage period with statistically similar values recorded during most of the storage period. Maximum color values were recorded in control during most of the storage period with the lowest percent decrease (6.1\%) estimated on $180^{\text {th }}$ day storage. In contrast, the percent 
decrease in L-value remained higher in aloe vera coated chips with utmost decline observed in 30\% concentration (10.7\%) during the same storage time. In terms of L-value, 10 and 20\% AV remained statistically same with an overall $8.7 \%$ decline in each treatment at the end of storage period (Figure 3).

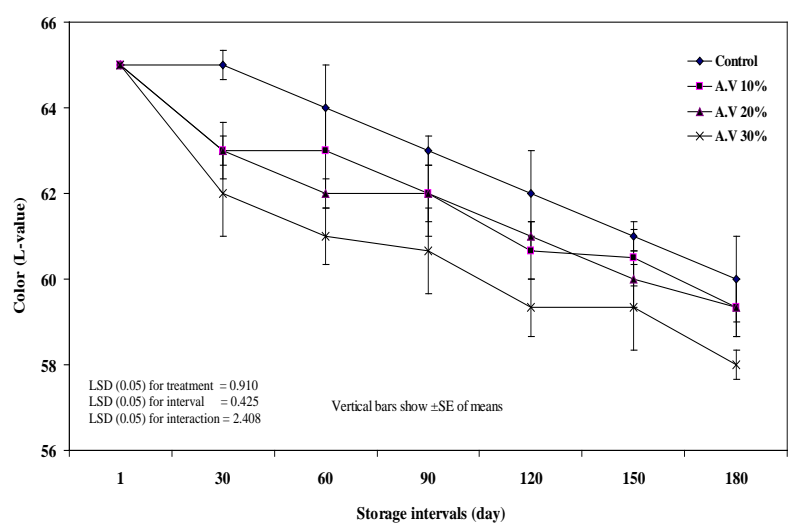

Figure 3. Comparison of chip color in response to aloe vera coatings on potato chips

L-values correspond to the lightness of chip color which is primarily associated with consumer acceptance [26]. Coatings are known to decrease the L- value in the processed product due to the formation of opaque layer. In the present study, CCL values were affected by different coating applications and decreased with the increase in the applied concentrations. For instance, chips coated with 10 and 20\% AV retained higher L-values than those coated with 30\% AV concentration. Similar results were reported by Khalil [23] who found decreased color values in French fries due to calcium chloride application with increased concentration. Our results showed that increase in AV concentration up to 10 and $20 \%$ maintained appreciable L-value up to some threshold level, however considerable decline in AV 30\% was noticed as a consequence of excess application. These results were comparable with the findings of Khalil [23]. Retention of acceptable L-value in aloe vera coated chips (10, 20\%) might be considered as a result of reduced acrylamide formation [9] in processed product. Similar observations regarding reduction of acrylamide contents as results of pre processing coating applications has been reported by Vattem and Shetty [27]. The steady decline in L-values in all the treatments along the storage intervals might be attributed to their increased reducing sugar contents Blenkinsop [28].

\subsection{Chip Crispiness}

Chip crispiness scores showed initial increase till the mid storage followed by gradual decline at the end. The effect of treatments, storage intervals and their interaction was found significant $(\alpha-0.05)$ for the chip crispiness. Appreciable CCR scores were recorded in low and moderate AV coatings during the storage period. 20\% AV concentration presented best CCR scores throughout the storage period, however remained statistically similar to $10 \%$ during the whole storage periods. Significant $(\alpha-0.05)$ decline in CCR scores was observed in 30\% application during the last month of storage (Figure 4).

Potato chip texture is often described as crispiness which is an important sensorial attribute for consumer's appreciations. Eminent crispy structure develops in potato chip due to the hardening of chip crust during frying at elevated temperatures [29]. Starch and proto pectin are the most imperative chemical components contributing to quality chip texture during processing [18]. The textural attributes of chips are reported to be affected by the application of different coatings due to modification of surface properties [6]. Formation of uniform coating on the chip surface is essential to impart even textural attributes. Application of AV 20\% presented remarkable chip texture during frying which might be due to the gelatinization of surface starch while inferior CCR scores were quantified in AV 30\% which might be due to their excessive moisture retention, which subsequently caused sogginess during processing [2]. Superior maintenance of textural attributes with acceptable scores due to hydrocolloid coating at different concentrations has also been reported by Kita [18] and Garcia et al. [8] previously.

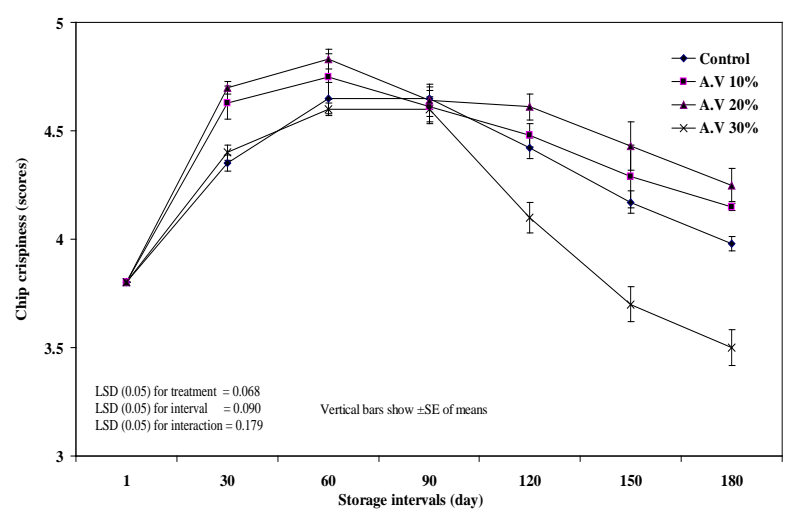

Figure 4. Comparison of chip crispiness in response to aloe vera coatings on potato chips

\subsection{Chip Flavor}

Chip flavor scores in general illustrated gradual initial increase followed by decline at the end of storage period. The effect of treatments, storage intervals and their interaction was found significant for the chip flavor ( $\alpha$ 0.05). Significant decline in CFL scores was observed in AV $30 \%$ after mid storage period with minimum scores (3.10/5.00) recorded on $180^{\text {th }}$ day. 10 and $20 \% \mathrm{AV}$ coatings maintained appreciable flavor at different storage intervals with final values of 3.74/5.00 and 3.78/5.00 respectively estimated at their ends. Control retained maximum flavor throughout the storage period with 4.04/5.00 CFL scores identified at the end of storage period (Figure 5).

Flavor is the sensory impression of food detected by the blend of taste and smell senses. It is the overall resultant impression derived by the taste buds in mouth and aroma detected by olfactory epithelium in nose. Flavor evolution in potato chips is primarily attributed to the oil uptake and corresponding volatile formations during thermal processing [30]. In general hydrocolloid coatings presented lower CFL scores as compared to control due to reduced oil uptake. Maximum CFL scores were estimated in control during most of the storage period which might be attributed to their high fat absorption during processing. Garcia et al. [8] evaluated the efficiency of different cellulose based edible coatings in reduced oil absorption during processing. He concluded that the combination of $1 \%$ methyl cellulose and $0.5 \%$ sorbitol proved to be most 
efficient coating for fat reduction and flavor retention in potato chips. In the present study, AV coatings maintained appreciable CFL scores of the processed chips except in $30 \%$ application which is also of great significance because of no coating based bitter flavor was identified by the judges during the evaluation. AV 30\% presented lowest flavor scores which might be due to AV associated bitter after taste and increased moisture contents in the processed chips.

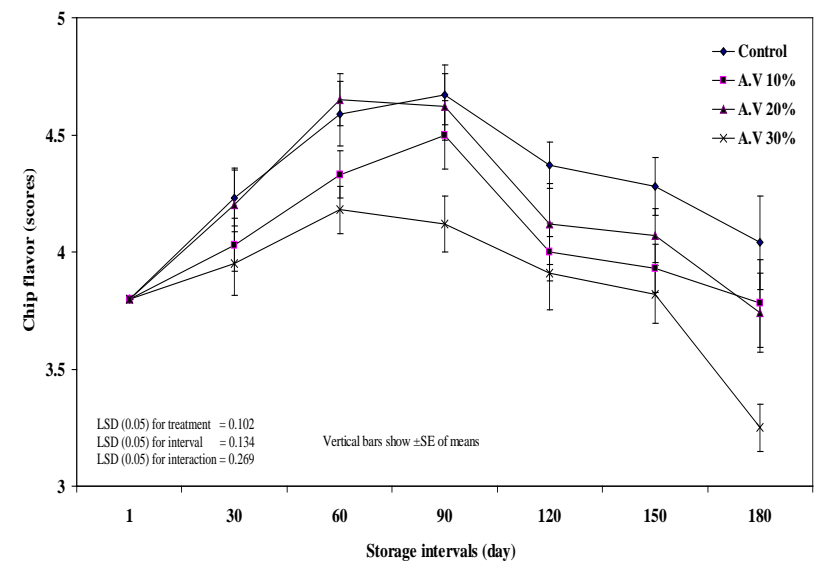

Figure 5. Comparison of chip flavor in response to aloe vera coatings on potato chips

\section{Conclusion}

This paper has evaluated the influence of AV gel concentrations on quality attributes of stored potato chips. $20 \%$ aloe vera concentration was found the most effective coating due to moderate moisture retention and lower oil contents in the processed chips. Although the color difference was detected between coated and control samples, however $20 \%$ concentration retained appreciable color and textural scores in the end product. Plant based edible coating for the preparation of low caloric processed products is required to address health, economic and ethical issues. Therefore, the current investigation revealed $20 \%$ AV gel concentration as a best choice to produce low caloric HALAL (permissible food in Islamic jurisprudence) processed product. The outcomes of the present research might be utilized by small as well as large scale processors to produce potato chips of low caloric value with higher sensory quality.

\section{References}

[1] Gamble MH, Rice P. The effect of slice thickness on potato crisp yield and composition. University of Technology, Loughborough, Leicestershire LE11 3TU, UK. 1988.

[2] Pedreschi F, Moyano P. Effect of pre-drying on texture and oiluptake of potato chips. LWT-Food Science and Technology, 2005; 38: 599-604.

[3] Mehta U, Swinburn, B. A review of factor effecting fat absorption in hot chips. Crit Rev Food Sci Nutr, 2001; 41: 133-154.

[4] Berry SK, Sehgal RC, Kalra CL. Comparative oil uptake by potato chips during frying under different conditions. Journal of Food Science and Technology, 1999; 36: 519-521.

[5] Mellema M. Mechanism and reduction of fat uptake in deep-fat fried foods. Trends in Food Science \& Technology, 2003; 14: 364373.
[6] Williams R, Mittal GS. Water and fat transfer properties of polysaccharide films on fried pastry mix. LWT-Food Science Technology, 1999; 32: 440-445.

[7] Mallikarjunan P, Chinnan MS, Balasubramaniam VM, Phillips RD. Edible coatings for deep-fat frying of starchy products. LWTFood Science and Technology, 1997; 30: 709-714.

[8] Garcia MA, Ferrero C, Bertola N, Martino M, Zaritzky N. Edible coatings from cellulose derivatives to reduce oil uptake in fried products. Innovative Food Science \& Emerging Technology, 2002; 3: 391-397.

[9] Fiselier K, Grob K, Pfefferle A. Brown potato croquettes low in acrylamide by coating with egg/breadcrumbs. European Food Research \& Technology, 2004; 219(2): 111-115.

[10] Albert S, Mittal GS. Comparative evaluation of edible coatings to reduce fat uptake in a deep-fried cereal product. Food Research International, 2002; 35: 445-458.

[11] Eshun K, He Q. Aloe vera: a valuable ingredient for the food, pharmaceutical and cosmetic industries a review. Critical Reviews in Food Science \& Nutrition, 2004; 44: 91-96.

[12] Reynolds T, Dweck AC. Aloe vera leaf gel: a review update. Journal of Ethnopharmacology, 1999; 68:3-37.

[13] Aburjai T, Natsheh FM. Plants used in cosmetics. Phytotherapy Research, 2003; 17: 987-1000.

[14] Moore ED, Macanalley BH. A drink containing mucilaginous polysaccharides and its preparation. US Patent, 1995; 5: 443-830.

[15] Martinez RD, Alburquerque N, Valverde J, Guillein M, Castillo FS, Valero D. Postharvest sweet cherry quality and safety maintenance by Aloe Vera treatment: a new edible coating. Post harvest Biology \& Technology, 2006; 39: 93-100.

[16] Valverde JM, Valero D, Omero DM, Guillean F, Castillo S, Serrano M. Novel edible coating based on aloe vera gel to maintain table grape quality and safety. Journal of Agriculture \& Food Chemistry, 2005; 53: 7807-7813.

[17] AOAC. Official Methods of Analysis. Association of Analytical Chemists. 15 ${ }^{\text {th }}$ Ed. Virginia 22201, Arlington, USA. 2005.

[18] Kita A. The influence of potato chemical composition on crisp texture. Food Chemistry, 2002; 76: 173-179.

[19] Steel RD, Torrie JH, Dickey D. Principle and Procedure of Statistics. A Biometrical approach: $3^{\text {rd }}$ Ed. McGraw-Hills Book Co. Inc. New York, 1997.

[20] Huse HL, Mallikarjunan P, Chinnan MS, Hung YC, Phillips RD. Edible coatings for reducing oil uptake in production of akara (deep-fat frying of cowpea paste). Journal of Food Processing \& Preservation, 1998; 22: 155-165.

[21] Garmakhany AD, Mirzaei H, Nejad MK, Maghsudlo Y. Study of oil uptake and some quality attributes of potato chips affected by hydrocolloids. European Journal of Lipid Science \& Technology, 2008; 110: 1045-1049.

[22] Aguilera JM, Gloria-Hernandez H. Oil absorption during frying of frozen par-fried potato. Journal of Food Science, 2000; 65: 476479.

[23] Khalil AH. Quality of French fried potatoes as influenced by coating with hydrocolloids. Food Chemistry, 1999; 66: 201.

[24] Rimac BS, Lelas V, Rade D, Simundic B. Decreasing of oil absorption in potato strips during deep fat frying. Journal of Food Engineering, 2004; 64: 237-241.

[25] Hagenimana V, Karuri EG, Oyunga MA. Oil content in fried processed sweet potato products. Journal Food Processing \& Preservation, 1998; 22: 123-137.

[26] Mendoza F, Dejmek P, Aguilera JM. Colour and image texture analysis in classification of commercial potato chips. Food Research International, 2007; 40: 1146-1154.

[27] Vattem DA, Shetty K. Acrylamide in food: a model for mechanism of formation and its reduction. Innovative Food Science \& Emerging Technologies, 2003; 4: 331-338.

[28] Blenkinsop RW, Copp LJ, Yada RY, Marangoni, AG. Changes in compositional parameters of potato (Solanum tuberosum) during low-temperature storage and their relationship to chip processing quality. Journal of Agricultural Food Chemistry, 2002; 50: 45454553.

[29] Pedreschi F, Aguilera JM, Pyle L. Textural characterization and kinetics of potato strips during frying. Journal of Food Sciences, 2001; 66: 314-318.

[30] Martin FL, Ames JM. Formation of Strecker aldehydes and pyrazines in a fried potato model system. Journal of Agricultural Food Chemistry, 2001; 49: 3885-3892. 